\title{
Controlling aggregation of non-ionic surfactants using mixed glycol media
}

\author{
Caroline Seguin $^{a}$, Julian Eastoe ${ }^{*}$, Richard K. Heenan ${ }^{b}$ and Isabelle Grillo ${ }^{c}$ \\ ${ }^{\text {a }}$ School of Chemistry, University of Bristol, Bristol, BS8 1TS, UK. \\ ${ }^{b}$ ISIS-CCLRC, Rutherford Appleton Laboratory, Chilton, Oxon, OX11 0QX, UK. \\ 'Institut Max-von-Laue-Paul-Langevin, BP 156-X, F-38042 Grenoble Cedex, France.
}

\section{Supporting Information}

\section{SANS}

\section{Materials and Methods}

Fully deuterated ethylene and propylene glycol were obtained from Qmx laboratory (CDN isotopes).

SANS experiments were carried out at the time-of-flight LOQ instrument at ISIS, UK where incident wavelengths are $2.2 \leq \lambda \leq 10 \AA$, resulting in an effective $Q$ range of $0.009 \rightarrow 0.249 \AA^{-1}$, or at the D22 diffractometer at ILL (Grenoble, France) using a neutron wavelength of $\lambda=10 \AA$ at two different detector distances to cover a $Q$ range of $0.0024 \rightarrow 0.37 \AA^{-1}$. Absolute intensities for $\mathrm{I}(\mathrm{Q})\left(\mathrm{cm}^{-1}\right)$ were determined to within $\pm 5 \%$ by measuring the incoherent scattering from $1 \mathrm{~mm}$ of $\mathrm{H}_{2} \mathrm{O}$ at ILL, whereas at ISIS a partially deuterated polymer standard was employed. Neutron counts accumulated over periods of 40 min provided sufficient statistical quality; a run for a fixed incident neutron monitor count was allowed for transmissions. Following standard procedures, raw data were treated to yield normalized scattering intensities $\mathrm{I}(Q)$ in $\mathrm{cm}^{-1}$, where the momentum transfer $Q=(4 \pi / \lambda) \sin (\theta / 2)$ and $\theta$ is the scattering angle $\left(<7^{\circ}\right)$. Scattering data were also corrected for wavelength-dependent transmission factors, as well as cell, background, and any hydrocarbon incoherent scattering. 
Sample preparation

For each surfactant/solvent combination, a $10 \mathrm{wt} \%$ solution was prepared. After these concentrated samples had been run, they were diluted by mass to yield $5 \mathrm{wt} \%$ solutions. Samples were held in $2 \mathrm{~mm}$ path-length Hellma quartz cells and thermostated at $25\left( \pm 1^{\circ} \mathrm{C}\right)$.

\section{Model Fitting}

SANS data were analyzed using standard Guinier limiting laws, and the multimodel FISH fitting program [1, 2], which is based upon an iterative least-squares algorithm. This program allowed for various common scattering laws to be tested, the best structural parameters to be obtained and also a measure of the fit residuals (sum of weighed squared errors SWSE). An approximate $Q$ resolution function was also included. The eventual model was not arrived at randomly; instead, a strategy of eliminations was used, taking into account the general form of the scattering curves (linear and logarithmic scale) and also results from limiting law Guinier analyses to test for common form factors (spheres, cylinders, discs).

All models included a form factor $P(Q)$ and an effective hard-sphere excluded volume structure factor $S(Q)$. Because the volume fraction of the micelles is unknown, and changes dramatically as a function of solvent type, the effective volume fraction $\phi_{\text {hs }}$ was allowed to float: however, it was always less than the total volume fraction of added surfactant. In all cases the $S(Q)$ functions were only weak (non-charged dilute systems) accounting for at worst $10 \%$ of the signal intensity, but typically $5 \%$. This approach, and the limitations, has been described elsewhere [1]. In other words, the $S(Q)$ contributions were only minimal, and entirely effective, used to improve the fits marginally from the pure form factors, which dominated the SANS signals. This can be seen by inspection of the data presented in the main paper, and here in supporting information: there is an obvious absence of any $S(Q)$ interaction peaks. In all cases the $S(Q)$ contributions were weak, and the main scattering was from form factor-type signals. Therefore, the clear evolutions of SANS intensities can be ascribed to overriding changes in the micellar shape (PQ)) and extent of aggregation (intensity).

The first form factor tested was for Schultz polydisperse spheres [1]. This did not give good statistical fits or reasonable parameters. On the other hand, for an ellipsoid core-shell model and rod core-shell form factor, both gave physically realistic 
fit parameters and good quality statistical fits were obtained. The main reasons for introducing an external shell of thickness $t$ was to account for the possibility of varying extents of solvation of the ethylene oxide groups as a function of solvent type, and also to improve the fit quality.

In the ellipsoid and rod core-shell model, the general scattering law [2] and the form factor $P(Q)$ are:

$$
\begin{gathered}
I(Q)=n_{p}\left[\frac{4}{3} \pi\left(r_{1}+t\right)^{2}\left(r_{2}+t\right)\right]^{2}\left(\rho_{\text {shell }}-\rho_{\text {solvent }}\right)^{2} P(Q) S(Q) \\
P(Q, \mu)=\left(3 \frac{j_{1}\left(u_{2}\right)}{u_{2}}+\left(\frac{\rho_{\text {core }}-\rho_{\text {shell }}}{\rho_{\text {shell }}-\rho_{\text {solvent }}} \times \frac{r_{2}^{3}}{\left(r_{1}+t\right)^{2}\left(r_{2}+t\right)}\right) \times 3 \frac{j_{1}\left(u_{1}\right)}{u_{1}}\right)^{2}
\end{gathered}
$$

In the above equations $r_{1}$ and $r_{2}$ are principal ellipsoid axes, the scattering length densities (SLD's) $\rho$ of the micellar internal core, outer shell and external solvent are defined in subscripts, $\mu$ is the cosine of the angle between the axis of revolution and the scattering vector $Q$. Other definitions are as follows, with $X$ the aspect ratio

$$
\begin{gathered}
u_{1}=Q r_{1}\left[X^{2} \mu^{2}+\left(1-\mu^{2}\right)\right]^{1 / 2} \\
u_{2}=Q\left[\left(r_{2}+t\right)^{2} \mu^{2}+\left(r_{1}+t\right)^{2}\left(1-\mu^{2}\right)\right]^{1 / 2} \\
j_{1}(u)=\frac{(\sin (u)-u \cdot \cos (u))}{u^{2}}
\end{gathered}
$$

For the ellipsoid model,

$$
\begin{gathered}
V_{\text {mic }}=\frac{4}{3} \pi \cdot R_{1}^{2} \cdot R_{2} \\
\mathrm{R}_{1}=\mathrm{r}_{1}+\mathrm{t} \\
\mathrm{R}_{2}=\mathrm{r}_{2}+\mathrm{t}
\end{gathered}
$$


where $R_{1}$ and $R_{2}$ are the overall radius of the micelle, $t$ is the shell thickness and $V_{\text {mic }}$, the effective micelle volume.

In the FISH modeling routine [2] the absolute scattering scale factor, $A_{\text {ellip, }}$ is given by the following expression

$$
A_{\text {ellip }}=n_{p} \cdot V_{\text {mic }}^{2} \cdot\left(\rho_{\text {shell }}-\rho_{\text {solvent }}\right)^{2}
$$

where $n_{p}$ is the number density of particles and $\rho$ is a scattering length density (SLD).

In the limit of $r_{2}>>r_{1}$ this ellipsoid scattering law tends to that for a cylinder [2], so that $r_{1}=R$ the cross-section radius and $2 r_{2}=L$, the cylinder length. In the analyses presented here, trial fits were conducted using the general ellipsoid model (above). If for any sample the fitted function was in the cylinder limit $r_{2}>>r_{1}$, then the cure was re-analyzed using the full cylinder form factor model as given in reference 2 . In this case the micellar volume $\mathrm{V}_{\text {mic }}$ is given by

$$
V_{\text {mic }}=\pi \cdot R^{2} \cdot L
$$

In the FISH program the rod scale factor, $A_{\text {rod }}\left(\mathrm{cm}^{-1}\right)$, is given by the following expression

$$
A_{\text {rod }}=10^{-24} \cdot \phi \cdot\left(\rho_{\text {shell }}-\rho_{\text {solvent }}\right)^{2}
$$

where $\phi$ is the volume fraction $\rho$ is the scattering length density (SLD), and the scalar $10^{-24}$ has the units of volume $\left(\mathrm{cm}^{3}\right)$.

Equation 12 was used to calculate the scattering length density of the different solvent mixtures (solvents $A$ and $B$ ).

$$
\rho_{\text {mixture }}=\phi_{\text {solventA }} x \rho_{\text {solventA }}+\left(1-\phi_{\text {solventA }}\right) x \rho_{\text {solventB }}
$$


where $\phi_{\text {solventA }}$ is the volume fraction of the solvent $A$ and $\rho_{\text {mixture }}\left(S L D_{\text {mixture }}\right), \rho_{\text {solvent }}$ and $\rho_{\text {solventB }}$ are the scattering length density of the mixture and the solvents $A$ and $B$, respectively. The values are listed in Table S1.

\begin{tabular}{ccccc}
\hline \multirow{2}{*}{ Solvent } & Mw $/\left(\mathrm{g} \mathrm{mol}^{-1}\right)$ & \multicolumn{2}{c}{ Volume fraction } & $\begin{array}{c}\text { SLD mixture } \\
\left(10^{-6} \AA^{-2}\right)\end{array}$ \\
\cline { 3 - 4 } & 68.1 & $\mathrm{~d}-\mathrm{PG}$ & $\mathrm{d}-\mathrm{EG}$ & 7.00 \\
10 & 69.7 & 0.12 & 1 & 7.00 \\
20 & 71.3 & 0.24 & 0.85 & 6.99 \\
40 & 74.5 & 0.46 & 0.74 & 6.99 \\
50 & 76.1 & 0.57 & 0.53 & 6.98 \\
70 & 79.3 & 0.76 & 0.43 & 6.97 \\
100 & 84.1 & 1 & 0.25 & 6.96 \\
\hline
\end{tabular}

Table S1 Solvent compositions, and calculated values for the solvent scattering length densities $\rho_{\text {mixture }}$ (SLD mixture) used in the analyses of SANS data by model fitting.

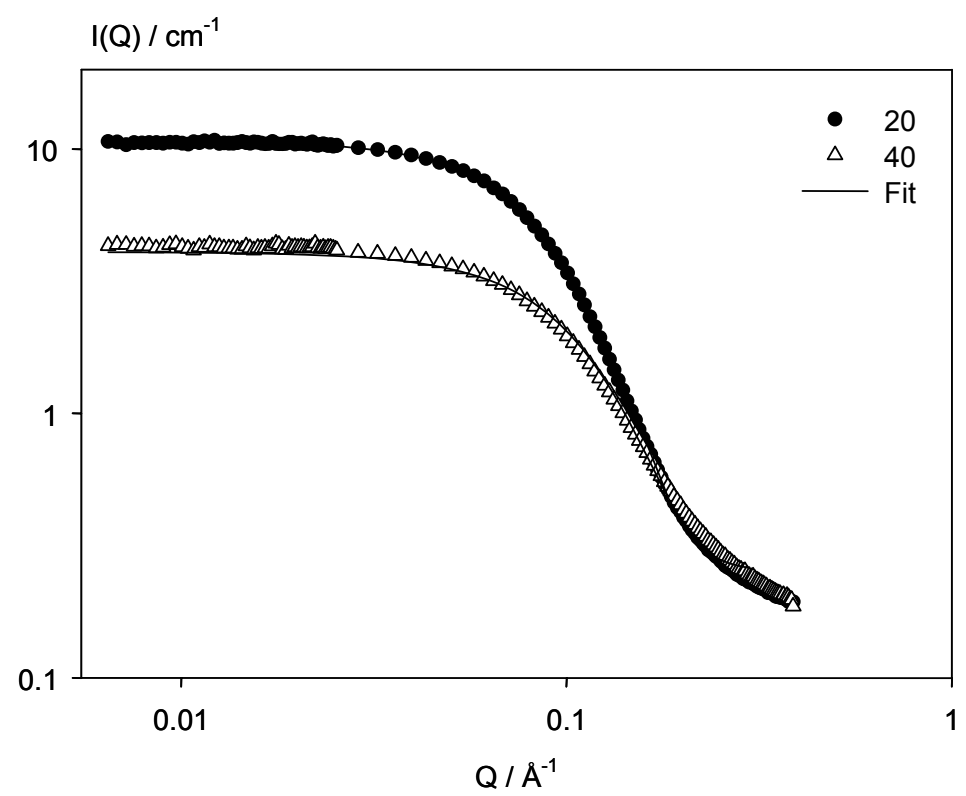

Figure S1 SANS data and model fits for 10 wt\% solutions of $\mathrm{C}_{12} \mathrm{E}_{8}$ in 20 and 40 mol\% d-PG in d-EG in log-log representation. The comparison is between the cylinder form factor (20 mol\% d-PG), and the oblate ellipsoid structure at $20 \mathrm{~mol} \% \mathrm{~d}$ $P G$ 


\section{SANS results}

Example SANS data and fits are given on Figures S1 and S2. The solid lines are fits and fitted parameters are listed in Table 1 in the main article.

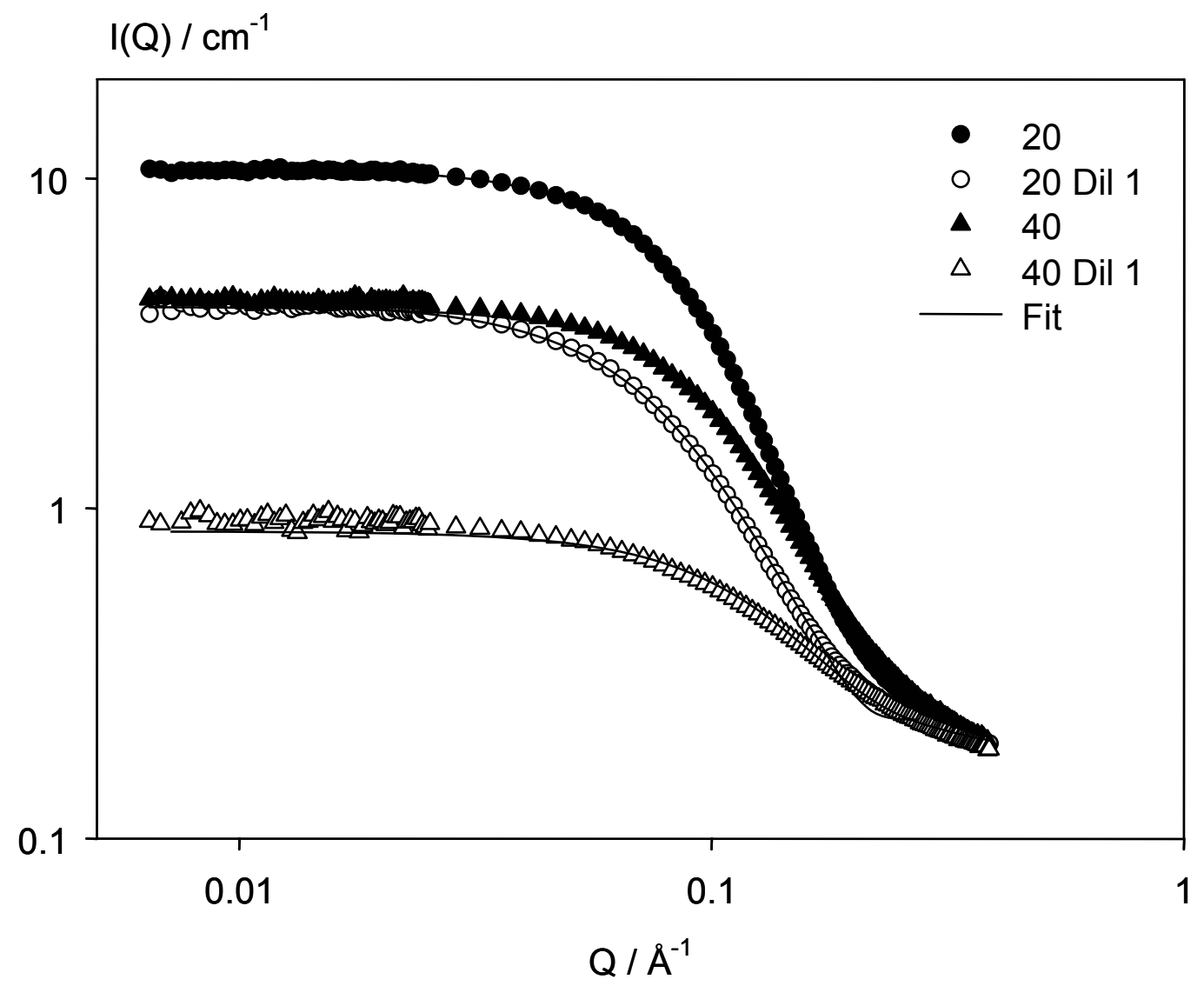

Figure S2 SANS curves for 10 and 5 wt\% (Dil 1) solutions of $\mathrm{C}_{12} \mathrm{E}_{8}$, in mixed EG: PG solvents at different mol\% of d-PG, at $25^{\circ} \mathrm{C}$. 


\begin{tabular}{ccccccc}
\hline Solvent & \multicolumn{2}{c}{ Radius / $\AA$} & \multicolumn{2}{c}{ Length / $\AA$} & \multicolumn{2}{c}{$\mathrm{V} /\left(\times 10^{4} \AA^{3}\right)$} \\
\hline & $10 \%$ & $5 \%$ & $10 \%$ & $5 \%$ & $10 \%$ & $5 \%$ \\
\hline 0 & 19 & 19 & 143 & 127 & 17 & 14 \\
10 & 19 & 19 & 84 & 100 & 9 & 11 \\
20 & 18 & 17 & 58 & 64 & 6 & 6 \\
\hline
\end{tabular}

Table S2a Parameters obtained by fitting SANS data to cylinder form factor from 5 and $10 \mathrm{wt} \%$ surfactant solutions of $\mathrm{C}_{12} \mathrm{E}_{8}$, in mixed EG: PG solvents at different mol\% of $d-P G$ at $25^{\circ} \mathrm{C} . V$ is the micellar volume. Uncertainties: Overall Radius $\pm 2 \AA$, Length $L \pm 10 \AA ; V \pm 3 \times 10^{3} \AA^{3}$.

\begin{tabular}{ccccccccc}
\hline Solvent & \multicolumn{2}{c}{$\begin{array}{r}\mathrm{r}_{1} /(\AA) \\
\text { (core radius) }\end{array}$} & \multicolumn{2}{c}{$\begin{array}{c}\mathrm{r}_{2} /(\AA) \\
\text { (core radius) }\end{array}$} & $\begin{array}{c}\mathrm{t} /(\AA) \\
\text { (thickness) }\end{array}$ & $\mathrm{V} /\left(\times 10^{4} \AA^{3}\right)$ \\
\hline & $10 \%$ & $5 \%$ & $10 \%$ & $5 \%$ & $10 \%$ & $5 \%$ & $10 \%$ & $5 \%$ \\
\hline 40 & 16 & 16 & 8 & 2 & 8 & 4 & 4 & 1 \\
50 & 16 & 16 & 5 & 2 & 5 & 3 & 2 & 1 \\
70 & 16 & - & 3 & - & 5 & - & 1 & - \\
100 & 16 & 16 & 1 & 1 & 3 & 3 & 1 & 1 \\
\hline
\end{tabular}

Table S2b Parameters obtained by fitting SANS data to core-shell oblate ellipsoid form factor from 5 and 10 wt $\%$ surfactant solutions of $\mathrm{C}_{12} \mathrm{E}_{8}$, in mixed EG: PG solvents at different mol\% of $d-P G$ at $25^{\circ} \mathrm{C}$. $V$ is the micellar volume. Uncertainties: $r_{1}, r_{2}, t \pm 2 \AA ; V_{\text {mic }} \pm 3 \times 10^{3} \AA^{3}$.

\section{References}

1. Eastoe, J.; Dupont, A.; Murray, M.; Martin, L.; Guittard, F.; Taffin de Givenchy, E.; Heenan, R.K. Langmuir, 2004, 20, 9953.

2. Heenan, R. K. FISH Data Analysis Program; Rutherford Appleton Laboratory; Report RAL-89129; CCLRC: Didcot, U.K., 1989. The program, a comprehensive manual describing the models and scattering laws, as well as appropriate accompanying references identifying the original sources of all these equations is available free from http://www.isis.rl.ac.uk/largescale/LOQ/loq.htm. Interested parties are kindly requested to first of all contact the author of this program at $\underline{\text { rkh@isis.rl.ac.uk. }}$ 